\title{
Internet-based adherence interventions for treatment of chronic disorders in adolescents
}

This article was published in the following Dove Press journal:

Adolescent Health, Medicine and Therapeutics

26 May 2015

Number of times this article has been viewed

\begin{abstract}
Alexandria M Bass'
Michael E Farhangian'

Steven R Feldman ${ }^{1-3}$

'Center for Dermatology Research, Department of Dermatology, Wake Forest School of Medicine, WinstonSalem, NC, USA; '2Department of Pathology, Wake Forest School of Medicine, Winston-Salem, NC, USA; ${ }^{3}$ Department of Public Health Sciences, Wake Forest School of Medicine, Winston-Salem, NC, USA
\end{abstract}

Background: Treatment adherence is a ubiquitous challenge in medicine, particularly in the adolescent population with chronic disorders. Web-based adherence interventions may be particularly useful in adolescents, due to their familiarity with and frequent use of the Internet.

Objective: To review web-based interventions used to improve adherence to medication in adolescent patients with chronic disorders.

Methods: A PubMed search was performed for full-text, English, clinical trials in adolescents using keywords "adherence" or "compliance", "Internet" or "web", and "treatment" from inception until November 2014. Articles were selected if they involved using the Internet to provide support to adolescents to help improve their adherence to treatment, excluding those focused on solely providing medical services through the Internet and articles focusing on preventative care, rather than treatment of an illness.

Results: Fourteen studies were found concentrating on chronic adolescent disorders. Interventions included online surveys, physician chat lines, monitoring programs, and interactive programs. All interventions experienced either greater improvement in adherence or another disease control measure or no statistically significant difference compared with the control group (in-clinic visits).

Limitations: Few clinical trials studying web-based interventions to improve adherence in adolescents were found. Due to not having one standard outcome measured in all of the studies, it was also difficult comparing the effectiveness of the interventions.

Conclusion: Web-based interventions play a role in improving adherence in adolescents with chronic disorders by being more time saving and cost effective compared with in-clinic visits. Further research focusing on adherence interventions using the Internet is necessary to determine the ideal ways of utilizing the Internet to improve adherence in adolescents with chronic disorders.

Keywords: web, compliance, teenagers, acne, diabetes, pediatrics

\section{Introduction}

Over the last few decades in medicine, advances in the research of chronic disorders have changed how physicians manage once fatal conditions in patients. This has led to millions of adolescents living with chronic disorders such as type 1 diabetes, asthma, and HIV due to improved screening, management, and treatment regimens available. ${ }^{1}$ However, treatment for these disorders often requires long-term cumbersome regimens consisting of multiple different medications taken multiple times a day, creating a hurdle to good adherence and high treatment failure rates.

Adherence is a challenge faced in many realms of medicine with an adherence rate of only $50 \%-60 \%$ across all patients. ${ }^{2}$ Poor adherence not only leads to poorer
Correspondence: Michael E Farhangia Center for Dermatology Research,

Department of Dermatology,

Wake Forest School of Medicine,

Medical Center Boulevard, Winston-

Salem, NC 27I57-107I, USA

$\mathrm{Tel}+\mathrm{I} 3367167740$

Fax +I 3367167732

Email mfarhang@wakehealth.edu 
treatment outcomes, but also leads to increases in health care spending due to emergency department visits, hospitalizations, and office visits. As of 2010, $83 \%$ of our health care resources were consumed by people with chronic disorders and non-adherence attributed to $33 \%-69 \%$ of all hospital admissions. ${ }^{3}$ Children and adolescents also account for a huge part of this problem with $50 \%-88 \%$ of those with chronic conditions being non-adherent to their medication. ${ }^{3}$

Today's adolescents have bonded with the Internet, with $27 \%$ of adolescents using the Internet for over 12 hours some days. ${ }^{4}$ Web-based applications could play a crucial role in supporting adolescent patients to improve their adherence to medication. Our objective was to review web-based interventions used to improve adherence to medication in adolescent patients with chronic disorders.

\section{Methods}

A PubMed search was performed using keywords "adherence" or "compliance" and "web" or "Internet" and "treatment" up until November 2014. Studies were filtered to include only full-text clinical trials performed with human subjects between the ages of 13-18 years old, in the English language. Articles were selected if they involved using the Internet or a web-based program to provide support to adolescents to help improve their adherence to treatment. Studies excluded were those that focused solely on providing medical services through the Internet and articles focusing on preventative care, rather than treatment of an illness. Twelve studies were found utilizing the database. In an effort to find more clinical trials, references of the included studies were screened and two additional articles were included (Figure 1). Only seven studies were found directly measuring adherence, so other disease outcomes, such as severity, quality of life, proper usage of treatments, knowledge about their disease, problemsolving capabilities, and limitations in daily activities were included. We classified the studies in this review based on the type of web-based intervention used to help improve adherence to treatment.

\section{Results}

Different web-based approaches such as emails containing surveys, chat lines, and online interactive programs

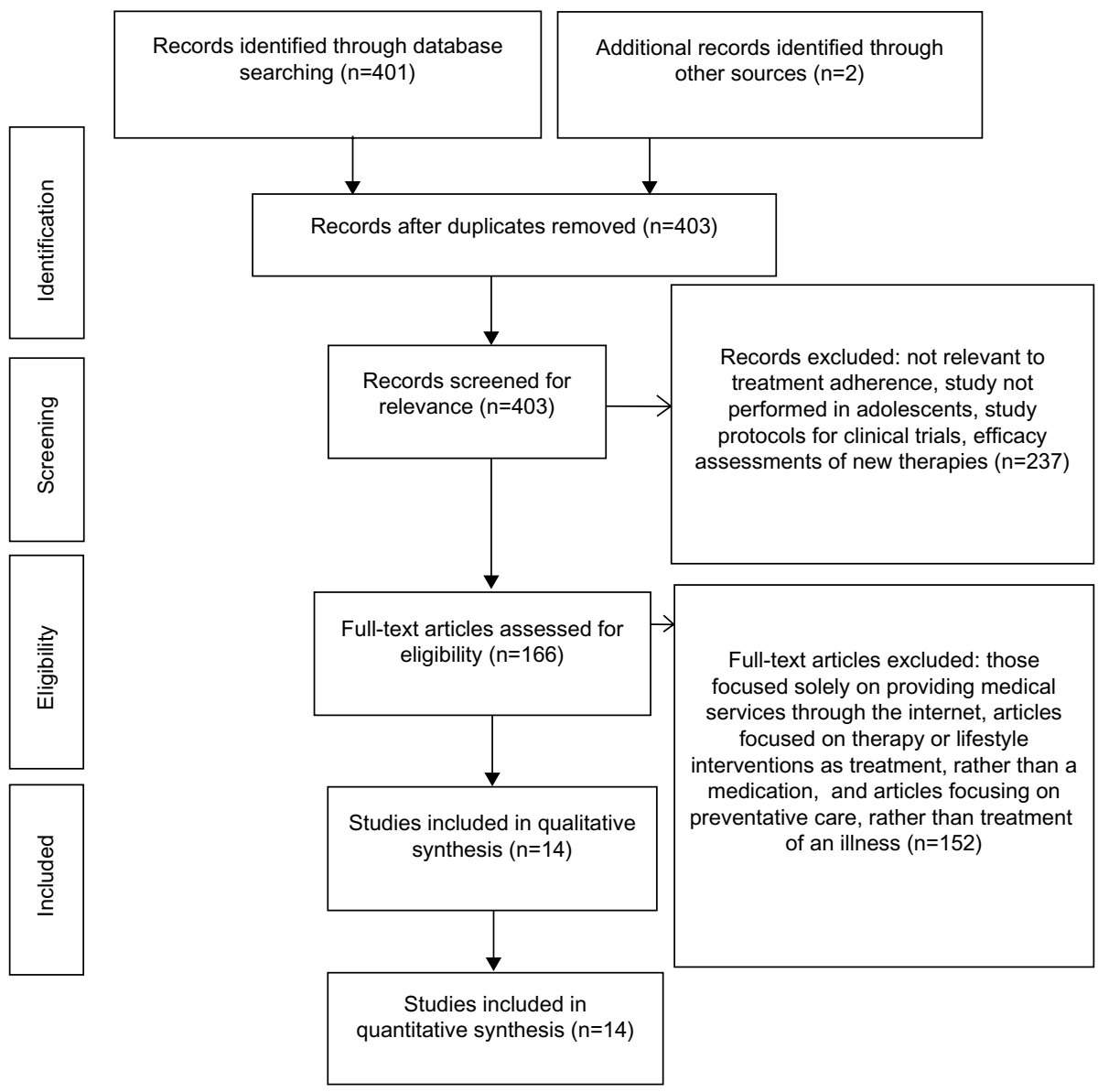

Figure I Preferred reporting items for systematic reviews and meta-analysis (PRISMA) 2009 flow chart. 
were evaluated to increase education and adherence of adolescents to their treatment (Table 1). The 14 studies focused on a variety of chronic adolescent disorders, such as acne,${ }^{5}$ type 1 diabetes, ${ }^{6-11}$ asthma, ${ }^{12-16}$ juvenile idiopathic arthritis, ${ }^{17}$ and HIV. ${ }^{18}$ The outcomes measured among the studies also varied with only a few studies directly measuring adherence. ${ }^{5,9,12,13,15,17}$ Other studies measured severity, ${ }^{6,7,14}$ quality of life, ${ }^{8,11}$ knowledge and self-efficacy, ${ }^{18}$ and/or problem-solving management ${ }^{10}$ instead of directly measuring adherence. It was assumed that improvements seen in these measures were due to increased adherence to treatment. The results are summarized according to type of intervention (Table 1).

\section{Web-based online survey}

Only one study was found examining the impact of the Internet in improving adherence in patients with acne. ${ }^{5}$ Adolescents, all using a benzoyl peroxide cream, received a weekly email containing a link to an online survey assessing their severity and treatment. At the end of the study, the intervention group had a greater overall adherence rate and a median adherence rate than the control group. The intervention group also experienced a greater improvement in the severity of their acne than the control group; however, these results were not statistically significant. Small sample size could have attributed to insignificant results in changes in severity. ${ }^{5}$

\section{Web-based monitoring programs}

Four studies examined monitoring system programs in adolescents with either type 1 diabetes or asthma., ${ }^{6,12,16}$ One study examined a website titled "Medtronic Carelink" for adolescents with type 1 diabetes. ${ }^{6}$ This website was an Internet-based blood glucose monitoring system that instructed participants in the intervention group to upload their glucose levels weekly over a 6-month period to a diabetes care team, who could make changes in the patient's regimen according to their data. After 6 months, hemoglobin $A_{1 c}$ $\left(\mathrm{HbA}_{1 \mathrm{c}}\right)$ in the intervention group declined and increased in the control group; however, these results were not statistically significant $(P=0.54)$. Out of 36 participants in the intervention group, 12 did not submit their blood glucose levels as instructed, leaving few compliant patients to retrieve results from during the study. ${ }^{6}$

Two similar studies by Chan et al in $2003^{12}$ and $2007^{13}$ also examined an Internet-based monitoring system to improve adherence to treatment, but in asthma patients. The differences were that in the 2003 study, the participants were required to record and upload their data (such as usage of their steroid inhaler, peak flow meter readings, and asthma symptom diary) to a website to be reviewed by a case manager. In the 2007 study, similar data were studied but instead of uploading it to a website, communication was via virtual visits for a year with the exception of three in-person visits. The control group for both studies received education and monitoring via traditional in-clinic scheduled appointments. After the completion of both studies, no significant differences were seen in adherence, quality of life, or severity. In the 2007 study, the intervention group experienced greater symptom-free days than the control group. ${ }^{12,13}$

Another study using online monitoring for adolescents with asthma was examined in 2012. ${ }^{16}$ Using a website, participants were encouraged to upload weekly parameters of asthma control, as well as have access to two online educational sessions and a personalized online asthma action plan. In conjunction with the website, patients continued to visit with their physician every 3-6 months as needed and for any exacerbations. The control group only had in-clinic visits every 3-6 months with their physician without access to the online monitoring program. After 1 year, no statistically significant differences were seen between the two groups in adherence, severity, quality of life, and knowledge. After 3 months, greater improvement was seen in severity and quality of life in the intervention group; however, these differences were not statistically significant by the completion of the study. ${ }^{16}$

\section{Physician chat line}

One study examined the efficacy of a weekly 1.5-hour physician mediated chat line for patients with type 1 diabetes. ${ }^{7}$ The topic of each chat, voted on by participants, focused on quality of life and metabolic control such as diabetes management and anxiety about the future. After 2 years, greater improvements in severity were appreciated in the intervention group; however, these results were not statistically significant $(P=0.056)$. Overall, all quality of life scores were higher in the intervention group with the greatest improvement found in the "Worries about Diabetes" scores.

\section{Online interactive programs}

Eight studies were found evaluating various web-based management programs intended for adolescents with chronic diseases such as Juvenile Idiopathic Arthritis (JIA), ${ }^{17}$ type 1 diabetes, ${ }^{8-11}$ HIV, ${ }^{18}$ and asthma. ${ }^{14,15}$ Most programs focused on education, management, and problem-solving strategies to improve adherence to treatment. Overall, the interactive 


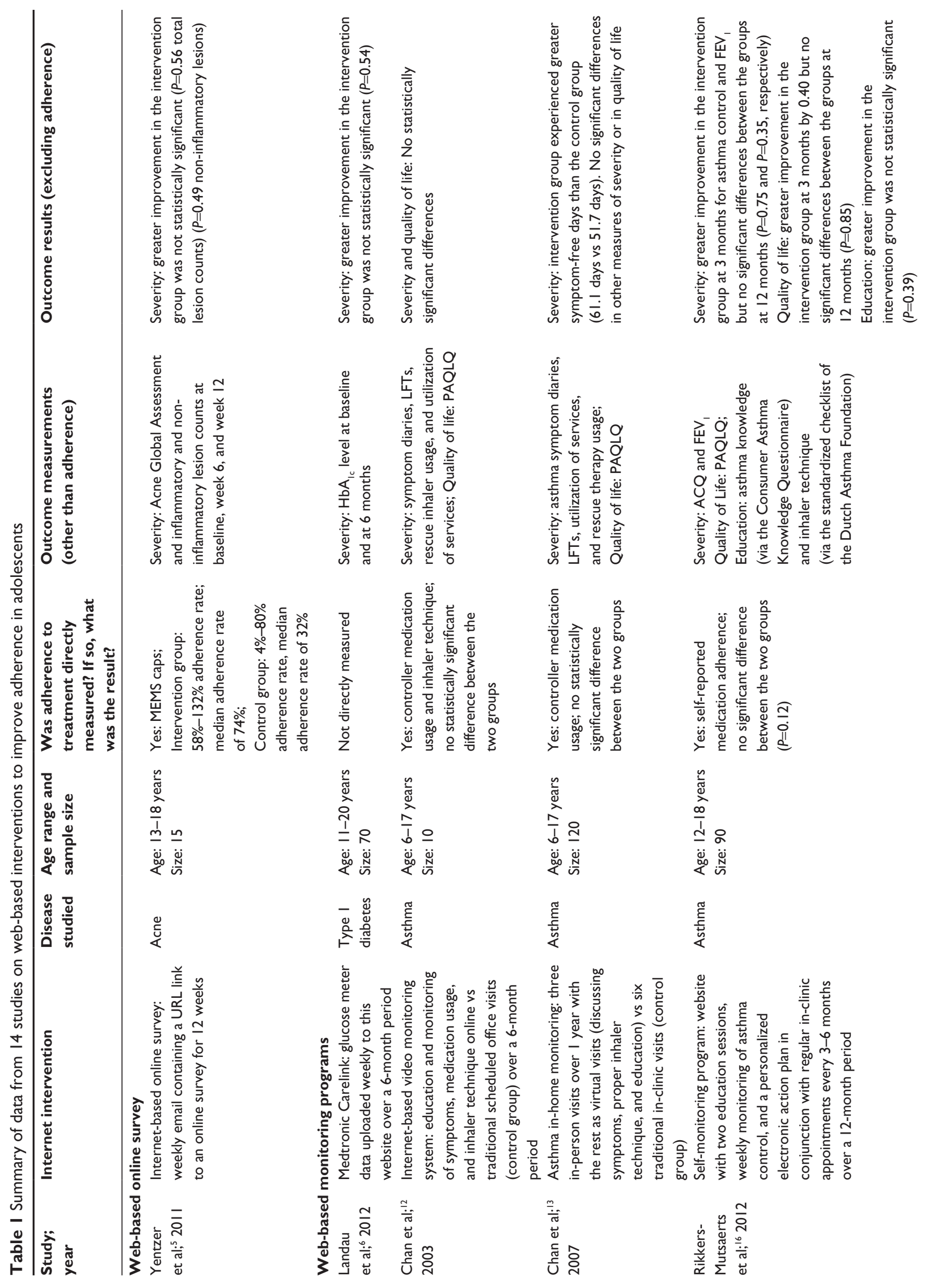



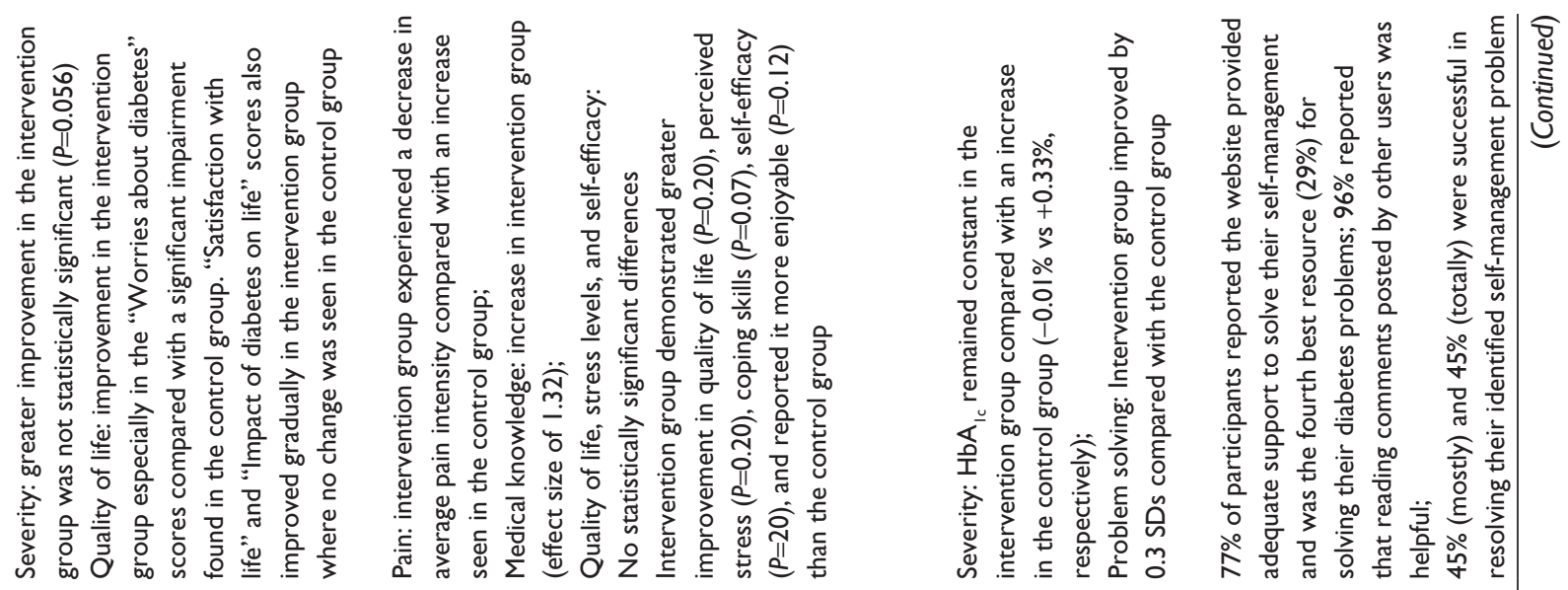

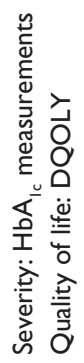
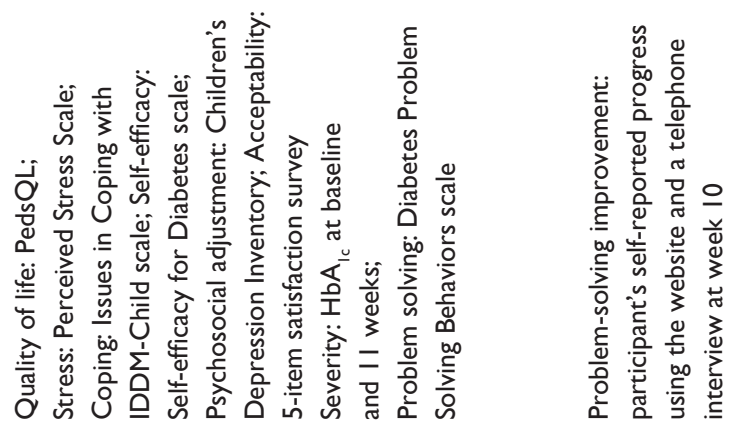

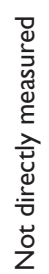
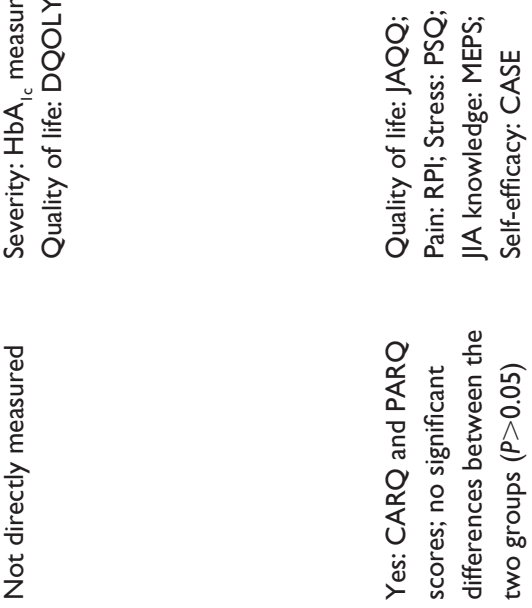

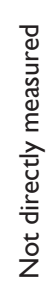

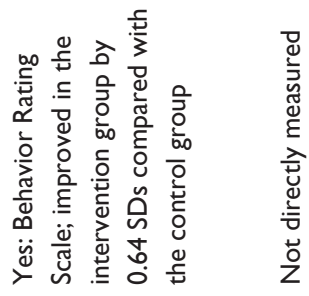

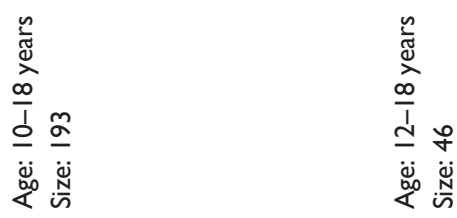

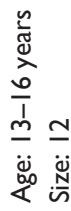

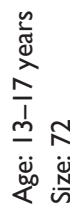

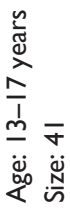

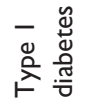

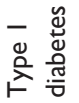

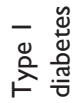

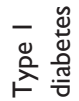
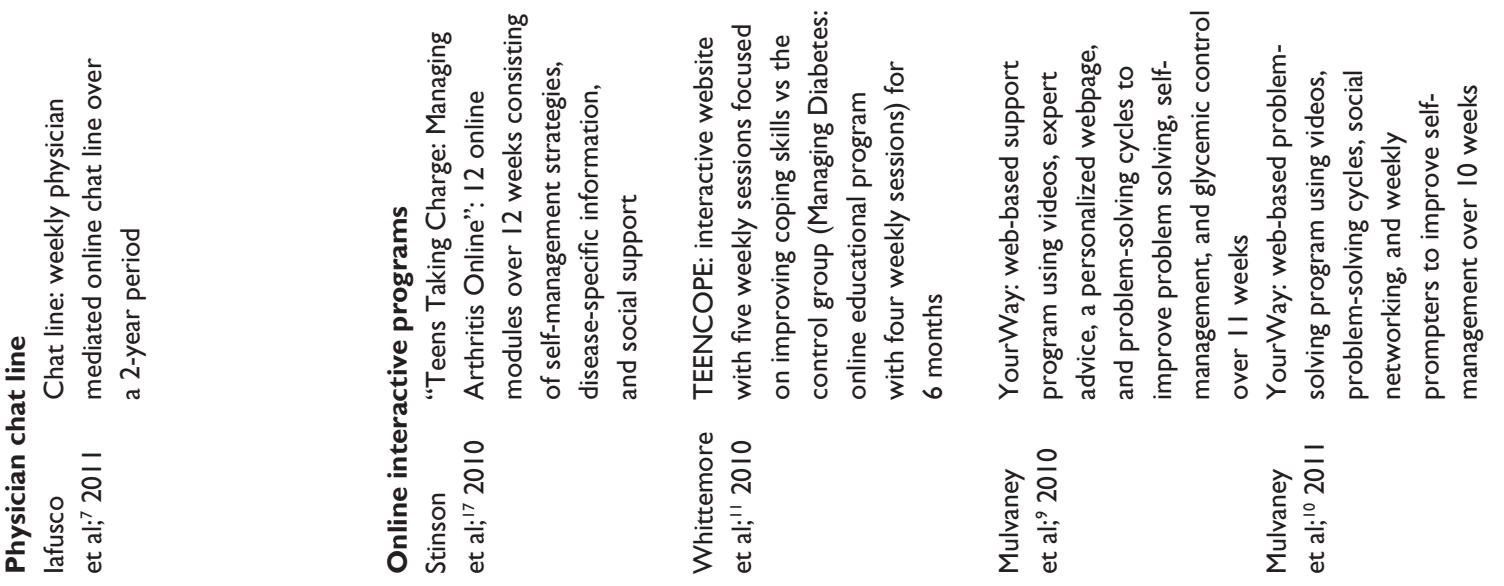


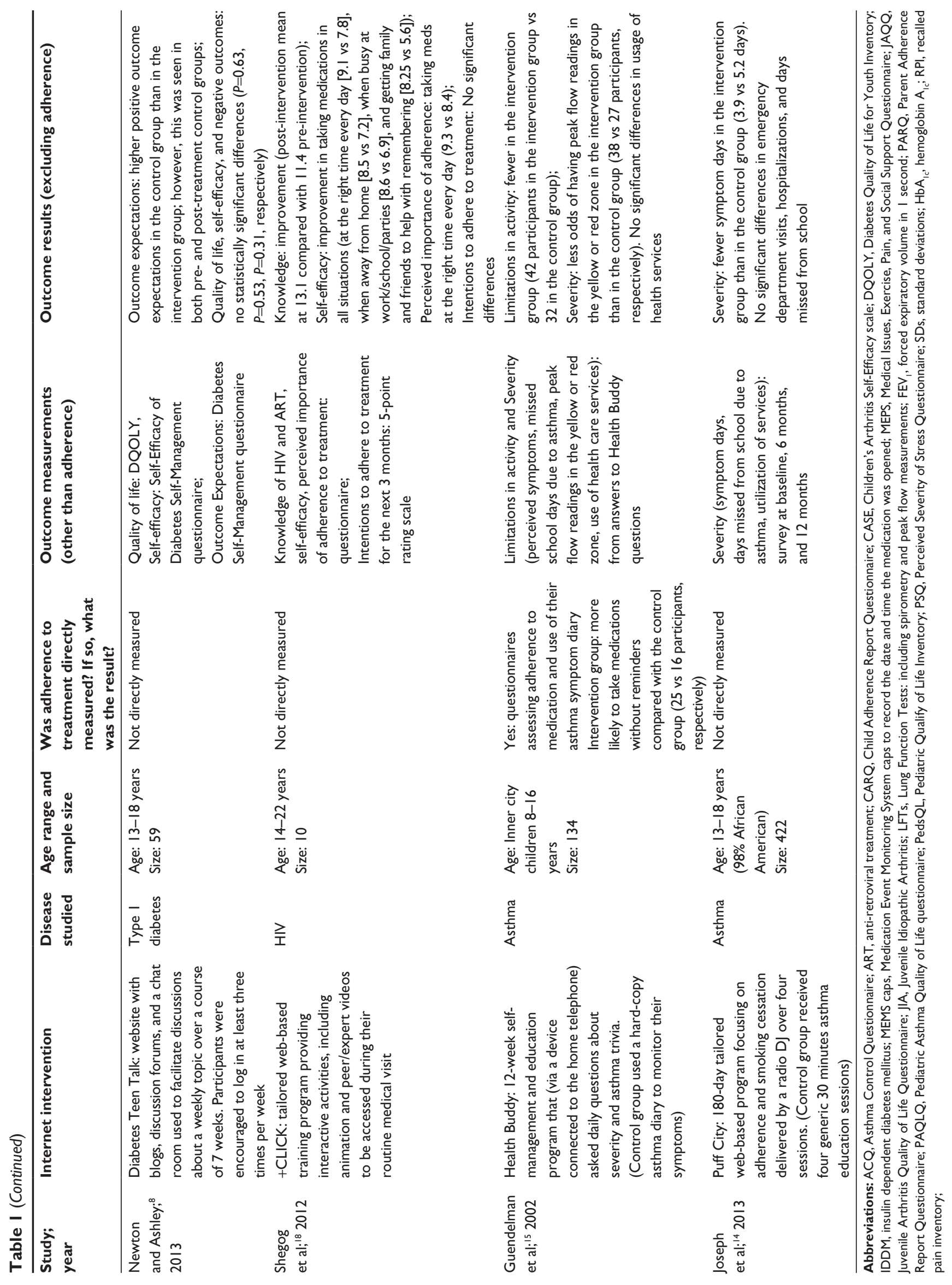


programs experienced the most improvements in measured outcomes compared with the control group. All eight studies had greater improvement in at least one measured outcome in the intervention group such as adherence, ${ }^{9,15}$ severity, ${ }^{9,14,15}$ quality of life, ${ }^{11}$ problem-solving management, ${ }^{9,10}$ coping, ${ }^{11}$ knowledge, ${ }^{17,18}$ pain, ${ }^{17}$ self-efficacy, ${ }^{11,18}$ and outcome expectations ${ }^{8}$ compared with the control group. The other outcomes measured in these studies had no statistically significant difference between the intervention group and the control group.

One program called "Teens Taking Charge: Managing Arthritis Online" was used for adolescents with JIA. ${ }^{17}$ This web-based self-management program focused on diagnosis, treatment, and management and was evaluated in 2010 to see whether it improved adherence to treatment. After 12 weeks, there was no significant difference between the two groups in adherence, quality of life, stress levels, or self-efficacy. The intervention group had a decrease in average pain intensity scores compared with the increase seen in the control group, in addition to an increase in medical knowledge. ${ }^{17}$ Limitations to this study were having a small sample size, which may have led to insignificant results.

Four interactive programs were used to improve adherence in adolescents with type 1 diabetes. ${ }^{8-11}$ One study evaluated an online interactive website focused on coping skills, called "TEENCOPE", vs the control group, an online educational website called "Managing Diabetes" over a 6-month period. ${ }^{11}$ At the completion of the study, the intervention group demonstrated greater improvement in quality of life, perceived stress, coping skills, self-efficacy, and reported it to be more enjoyable than the control group. ${ }^{11}$

Two studies, performed by Mulvaney et al in $2010^{9}$ and $2011,{ }^{10}$ focused on a self-management online program titled "YourWay", indicated for adolescents with type 1 diabetes. The program consisted of multimedia stories, problemsolving cycles, problem-solving expert advice, social networking, and weekly emails to encourage participation. The differences in the two studies were the outcomes measured. After 11 weeks of the 2010 study, adherence and problem solving were higher in the intervention group compared with the control group. Also, severity was maintained in the intervention group compared with worsening severity in the control group. ${ }^{9}$ The 2011 study examined the participants' opinions of the quality of self-management support, rather than adherence or severity. After 10 weeks, $77 \%$ of all of the participants felt that the website provided adequate support to solve their self-management problems. In addition, $45 \%$ (mostly) and 45\% (totally) were successful in resolving their identified self-management problem. ${ }^{10}$ Even though the 2011 study does not focus on improvements in quality of life, severity, or adherence, it provides common adolescent barriers to adherence and an additional way that these problems can be overcome.

"Diabetes Teen Talk" was the third interactive program found for adolescents with type 1 diabetes. ${ }^{8}$ This website consisted of three main areas: blogs, discussion forums, and a chat room used to facilitate discussion about a weekly topic over a course of 7 weeks. No significant difference was seen in quality of life between the two groups, self-efficacy, or negative outcome expectations. A significant difference was seen with the control group reporting a higher positive outcome expectation than individuals in the intervention group; however, this was also seen in both pre- and posttreatment control groups. However, the sample size may have been too small, limiting the study and leading to insignificant results. ${ }^{8}$

"+CLICK" was an online program intended to enhance adherence to treatment among HIV-positive youth based on motivational interviewing principles. ${ }^{18}$ The program was customized based on the patient's reported missed doses, blood counts, self-efficacy, psychosocial issues of perceived importance, and their goals regarding adherence. It provides interactive activities to be accessed during their routine office visit. At the end of the study, improvement was seen in knowledge about HIV and anti-retroviral therapy adherence at post-intervention compared with pre-intervention, in perceived importance of taking medications at the correct time every day, and in self-efficacy about taking their medications. No significant differences were appreciated in psychosocial variables and behavioral intentions to adhere to medications. Although these results do not provide direct information regarding improved adherence, the program may encourage patients to become more involved with their health and in facilitating discussion with health care providers. ${ }^{18}$

"Health Buddy", a program designed to improve asthma self-management in inner city children, was evaluated in $2002 .{ }^{15}$ The interactive 12 -week program was a device, connected to a home telephone, which was accessed daily. It was programmed to present ten questions pertaining to symptoms, peak flow readings, use of services, activity limitations, and asthma facts and trivia and then record the participant's answers. The intervention group reported greater improvements in most measures of severity (such as fewer limitations in activity and less odds of peak flow meter readings in the yellow or red zone) and adherence compared with the control group. No significant difference was appreciated between the two groups in usage of health services. ${ }^{15}$ 
In 2013, another Internet-based asthma prevention program was evaluated titled "Puff City". ${ }^{14}$ It was intended for 9th-12th grade students with asthma and focused on three behaviors: controller medication adherence, keeping an inhaler nearby, and smoking reduction/cessation. The program was divided into four sessions (15-30 minutes) over a 180-day period delivered by a radio DJ with tailored information presented to each teenager. No significant differences were appreciated between the two groups except for fewer symptom days were reported in the intervention group compared with the control group. ${ }^{14}$

\section{Discussion}

Several types of web-based adherence interventions for many chronic disorders were assessed in this review. Due to the variation in outcomes measured among the studies, the comparison between the interventions was rather difficult. However, common trends of effectiveness were seen with adherence and severity measures.

Out of the seven studies that directly measured adherence ${ }^{5,9,12,13,15-17}$ three of them had improvements in adherence in the intervention group ${ }^{5,9,15}$ with the other four having no significant differences between the intervention and control groups. ${ }^{12,13,16,17}$ The three with improvements were interventions using an online survey ${ }^{5}$ and two of the online interactive programs. ${ }^{9,15}$

Another outcome measured frequently was severity, being measured in all the studies except for five of the interactive program intervention studies. ${ }^{8,10,11,17,18}$ Greater improvements in severity in the intervention group compared with the control group were seen in four of the nine studies evaluating one of the online monitoring programs ${ }^{13}$ and three of the interactive programs..$^{9,14,15}$ No statistically significant differences were seen in the intervention using online surveys, ${ }^{5}$ three of the online monitoring programs, ${ }^{6,12,16}$ and the physician chat line. ${ }^{7}$ All of the online interactive programs that measured severity as an outcome saw significant improvement in some aspect of severity in the intervention group.

Overall, the web-based interventions reviewed were beneficial to patients. In studies where adherence or disease outcomes were measured, there were clear (albeit sometimes not statistically significant) improvements compared with traditional in-clinic appointments used in the control groups. The intervention groups experienced improvements in adherence and/or other aspects of their disease such as severity, quality of life, problem solving, coping, education, and pain levels. Further clinical trials performed with web-based interventions on improving adherence could provide more answers on their true effectiveness.

Intervention groups differed from the control groups in that they were participating in some form of additional communication, education, and/or monitoring in-between their in-clinic visits. These methods kept patients as active participants in managing their disease, which likely was a large contributor to why the patients in the intervention group experienced greater improvement. Maintaining accountability with patients using web-based interventions may be explained by a similar concept termed "white coat compliance". This phenomenon is when patients tend to increase their adherence to treatment around the time of follow-up appointments, possibly explaining why decreasing the time between follow-up appointments is an effective way to increase adherence to treatment. ${ }^{19}$ Allowing patients to log in online, complete a learning module, or upload data to a website instead of returning for weekly in-clinic follow-up appointments could provide insight to a more convenient method of maintaining adherence in patients.

In addition, even though the interventions reviewed were created to supplement traditional in-clinic visits, utilizing these interventions in lieu of office visits could provide a more time saving and cost-effective way of keeping up with patients to maintain adherence. ${ }^{20}$ Patients could schedule follow-up appointments farther apart and could avoid missing school/activities, the hassle of driving to the physician's office, waiting at the office, and paying for the office visit.

There were a couple of limitations to this review article. Most importantly, we found few clinical trials studying webbased interventions to improve adherence in adolescents. In addition, many of the studies did not directly measure adherence, so the assumption was made that improvements in other outcomes measured were due to improved adherence to treatment. Due to not having one standard outcome measured in all of the studies, it was also difficult to compare the effectiveness of the interventions.

\section{Conclusion}

Web-based interventions projected on improving adherence in adolescents may play a huge future role in the future of the treatment of chronic disorders. These interventions provide patients with a sense of accountability for managing their disorders in a more accessible way than in the past. Utilizing a source that so many adolescents already use on a daily basis could be the key to success in monitoring and treating patients effectively. Further research focusing on adherence 
interventions using the Internet is necessary to determine the ideal ways of implementing the Internet to improve adherence in adolescents with chronic disorders.

\section{Disclosure}

The Center for Dermatology Research is supported by an unrestricted educational grant from Galderma Laboratories, LP. Dr Steven R Feldman is a speaker for Janssen and Taro. He is a consultant and speaker for Galderma, Stiefel/ GlaxoSmithKline, Abbott Labs and Leo Pharma Inc. He has received grants from Galderma, Janssen, Abbott Labs, Amgen, Stiefel/GlaxoSmithKline, Celgene, and Anacor. He is a consultant for Amgen, Baxter, Caremark, Gerson Lehrman Group, Guidepoint Global, Hanall Pharmaceutical Co, Ltd, Kikaku, Lilly, Merck and Co, Inc., Merz Pharmaceuticals, Mylan, Novartis Pharmaceuticals, Pfizer Inc., Qurient, Suncare Research and Xenoport. He is on an advisory board for Pfizer Inc. He is the founder and holds stock in Causa Research and holds stock and is majority owner in Medical Quality Enhancement Corporation. He receives Royalties from UpToDate and Xlibris. Alexandria M Bass and Michael Farhangian report no conflicts of interest in this work.

\section{References}

1. Compas BE, Jaser SS, Dunn MJ, Rodriguez EM. Coping with chronic illness in childhood and adolescence. Annu Rev Clin Psychol. 2012;8:455-480.

2. Modi AC, Ingerski LM, Rausch JR, Glauser TA, Drotar D. White coat adherence over the first year of therapy in pediatric epilepsy. J Pediatr. 2012;161(4):695-699. e691.

3. McGrady ME, Hommel KA. Medication adherence and health care utilization in pediatric chronic illness: a systematic review. Pediatrics. 2013;132(4):730-740.

4. Kiatrungrit K, Hongsanguansri S. Cross-sectional study of use of electronic media by secondary school students in Bangkok, Thailand. Shanghai Arch Psychiatry. 2014;26(4):216-226.

5. Yentzer BA, Wood AA, Sagransky MJ, et al. An Internet-based survey and improvement of acne treatment outcomes. Arch Dermatol. 2011; 147(10):1223-1224.

6. Landau Z, Mazor-Aronovitch K, Boaz M, et al. The effectiveness of Internet-based blood glucose monitoring system on improving diabetes control in adolescents with type 1 diabetes. Pediatr Diabetes. 2012;13(2): 203-207.
7. Iafusco D, Galderisi A, Nocerino I, et al. Chat line for adolescents with type 1 diabetes: a useful tool to improve coping with diabetes: a 2-year follow-up study. Diabetes Technol Ther. 2011;13(5):551-555.

8. Newton KT, Ashley A. Pilot study of a web-based intervention for adolescents with type 1 diabetes. J Telemed Telecare. 2013;19(8): 443-449.

9. Mulvaney SA, Rothman RL, Wallston KA, Lybarger C, Dietrich MS An internet-based program to improve self-management in adolescents with type 1 diabetes. Diabetes Care. 2010;33(3):602-604.

10. Mulvaney SA, Rothman RL, Osborn CY, Lybarger C, Dietrich MS, Wallston KA. Self-management problem solving for adolescents with type 1 diabetes: intervention processes associated with an Internet program. Patient Educ Couns. 2011;85(2):140-142.

11. Whittemore R, Grey M, Lindemann E, Ambrosino J, Jaser S. Development of an Internet coping skills training program for teenagers with type 1 diabetes. Comput Inform Nurs. 2010;28(2):103-111.

12. Chan DS, Callahan CW, Sheets SJ, Moreno CN, Malone FJ. An Internetbased store-and-forward video home telehealth system for improving asthma outcomes in children. Am J Health Syst Pharm. 2003;60(19): 1976-1981.

13. Chan DS, Callahan CW, Hatch-Pigott VB, et al. Internet-based home monitoring and education of children with asthma is comparable to ideal office-based care: results of a 1-year asthma in-home monitoring trial. Pediatrics. 2007;119(3):569-578.

14. Joseph CL, Ownby DR, Havstad SL, et al. Evaluation of a web-based asthma management intervention program for urban teenagers: reaching the hard to reach. J Adolesc Health. 2013;52(4):419-426.

15. Guendelman S, Meade K, Benson M, Chen YQ, Samuels S. Improving asthma outcomes and self-management behaviors of inner-city children: a randomized trial of the Health Buddy interactive device and an asthma diary. Arch Pediatr Adolesc Med. 2002;156(2):114-120.

16. Rikkers-Mutsaerts ER, Winters AE, Bakker MJ, et al. Internet-based self-management compared with usual care in adolescents with asthma: a randomized controlled trial. Pediatr Pulmonol. 2012;47(12): 1170-1179.

17. Stinson JN, McGrath PJ, Hodnett ED, et al. An internet-based selfmanagement program with telephone support for adolescents with arthritis: a pilot randomized controlled trial. J Rheumatol. 2010;37(9): 1944-1952.

18. Shegog R, Markham CM, Leonard AD, Bui TC, Paul ME. "+CLICK”: pilot of a web-based training program to enhance ART adherence among HIV-positive youth. AIDS Care. 2012;24(3):310-318.

19. Feinstein AR. On white-coat effects and the electronic monitoring of compliance. Arch Intern Med. 1990;150(7):1377-1378.

20. van der Meer V, van den Hout WB, Bakker MJ, et al. Cost-effectiveness of Internet-based self-management compared with usual care in asthma. PLoS One. 2011;6(11):e27108.

\section{Publish your work in this journal}

Adolescent Health, Medicine and Therapeutics is an international, peer-reviewed, open access journal focusing on health, pathology, and treatment issues specific to the adolescent age group. All aspects of health maintenance, preventative measures and disease treatment interventions are addressed within the journal and practitioners from

\section{Dovepress}

all disciplines are invited to submit their work as well as healthcare researchers and patient support groups.. The manuscript management system is completely online and includes a very quick and fair peerreview system. Visit http://www.dovepress.com/testimonials.php to read real quotes from published authors. 